\title{
A novel duraplasty technique following fenestration of a massive lumbar arachnoid cyst in a patient with scoliosis: technical case report
}

\author{
Matthew T. Neal, MD, Randall J. Hlubek, MD, Alexander E. Ropper, MD, and U. Kumar Kakarla, MD \\ Department of Neurosurgery, Barrow Neurological Institute, St. Joseph's Hospital and Medical Center, Phoenix, Arizona
}

\begin{abstract}
When a dural defect is encountered during spine surgery, the dura mater must be reconstituted to minimize the occurrence of minor or major life-threatening sequelae. The neurosurgical literature lacks strategies for managing large dural defects encountered during surgery. The authors describe a 24 -year-old man who developed cauda equina syndrome secondary to altered CSF flow in a large thoracolumbar arachnoid cyst. Surgical decompression and fenestration of the arachnoid cyst were performed, and the large dural defect was treated using a multilayer closure with collagen matrix, titanium mesh, and methylmethacrylate. At his 24-month postoperative follow-up, the patient had recovered full strength in his legs, and his sensory deficits and sexual dysfunction had resolved. His incision had healed well, and there were no signs of pseudomeningocele. He had no additional positional headaches. The defect was managed effectively with this technique. Although this technique is not a first-line strategy for dural closure in the spine, it can be considered in challenging cases when large dural defects are not amenable to traditional closure techniques.
\end{abstract}

https://thejns.org/doi/abs/10.3171/2017.6.SPINE17155

KEY WORDS arachnoid cyst; cerebrospinal fluid; dural repair; duraplasty; durotomy; lumbar; methylmethacrylate; scoliosis; spine deformity; surgical technique

$\mathrm{D}$ UROTOMIES with CSF leakage are one of the most common complications of spine surgery? Without a watertight dural closure, minor complications such as transient headaches due to intracranial hypotension may result. More serious complications such as delayed neurological decline or infections, including meningitis, may also occur. ${ }^{2}$

Many strategies are available to repair a dural defect. Small defects that can easily be exposed and visualized can usually be repaired primarily with direct suturing. However, even with "routine" durotomies, intraoperative and postoperative management are not standardized. This variability in management is highlighted by a recent survey of 149 German neurosurgical departments. ${ }^{1}$ Suture technique, use of muscle patch or fibrin glue, and need for bed rest were inconsistent among those surveyed.

Many reports in the literature support the use of fibrin glue or hydrogel sealants to augment dural closure after intentional or incidental durotomies. ${ }^{3-5,9}$ However, the patients in these studies tended to have small dural defects, which can be sutured primarily, augmented with a patch graft, or reapproximated to minimize the size of the defect.

In this paper, we present the unique case of a young man with idiopathic scoliosis and an incidental, large, thoracolumbar arachnoid cyst. After scoliosis surgery, he developed delayed cauda equina syndrome, presumably related to the arachnoid cyst. During the operation to fenestrate the multicompartmental cyst, we noticed that the dural layer was extremely thin. The thick arachnoid cyst walls were compressing the nerve roots. After fenestration, a large area of the subarachnoid space had no dural coverage, and the visible dural edges were friable and could not be sutured. We describe a novel technique that we used to successfully manage the large dural defect in this patient. To our knowledge, no similar report exists in the literature.

\section{Case Report}

A 24-year-old man presented for evaluation of idiopathic scoliosis in 2014 (Fig. 1). The radiographic progression 


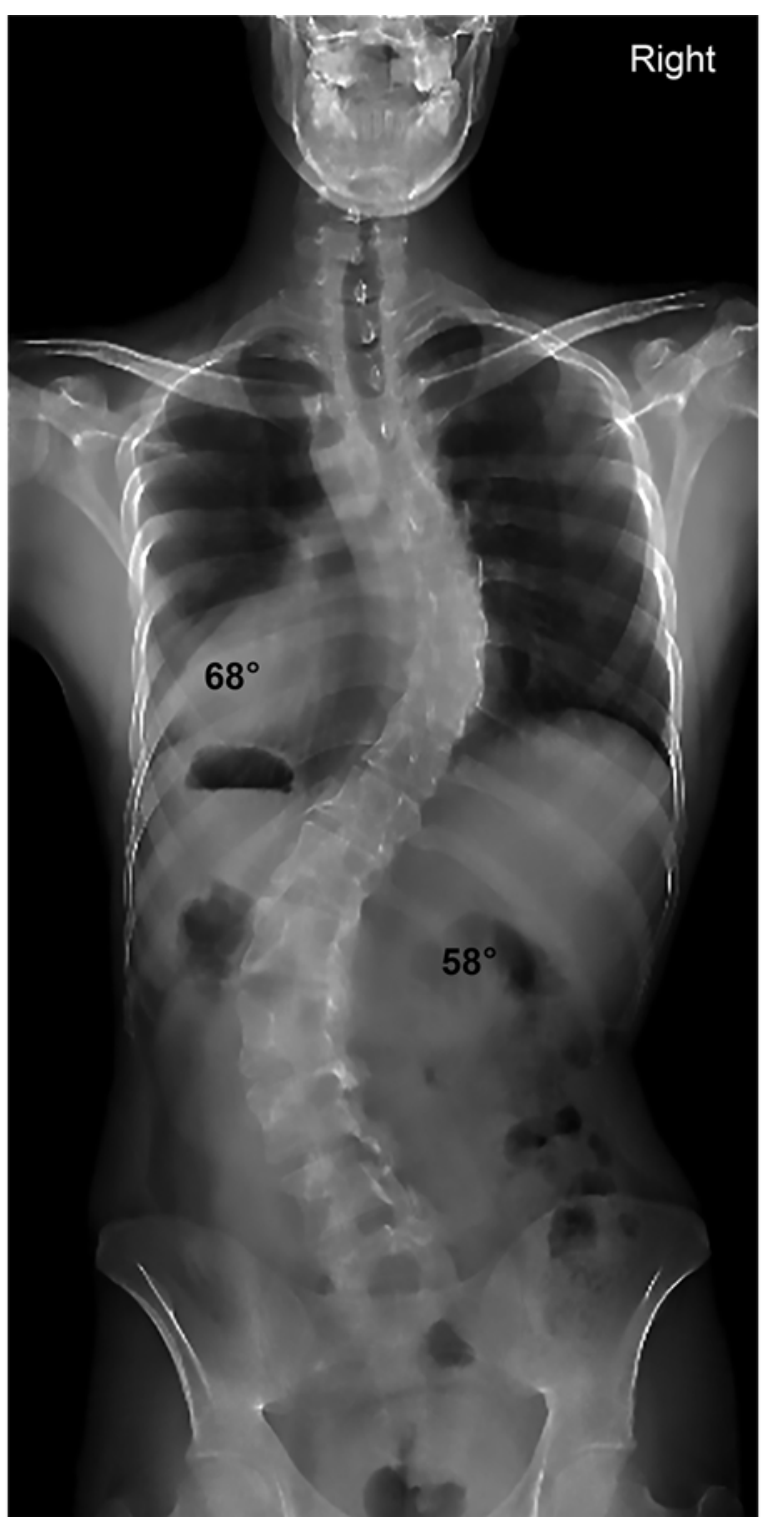

FIG. 1. Anteroposterior (AP) radiograph demonstrates scoliosis prior to first surgery. Reprinted from Ryan D (ed): Handbook of Neuroscience Nursing: Care of the Adult Neurosurgical Patient. New York: Thieme, 2017. Reprinted with permission from Barrow Neurological Institute, Phoenix, Arizona.

of his thoracic and lumbar curves, as well as his worsening back pain due to the lumbar hump, led us to recommend surgery to correct the spinal deformity. The patient was also noted to have a large arachnoid cyst spanning T-12 to L-4. However, he had no neurological deficits, and a CT myelogram demonstrated that contrast material communicated with the cyst (Fig. 2). Therefore, no plans were made to address the cyst during the operation. The patient underwent a posterior instrumented T3-L3 fusion (Fig. 3). Sagittal and coronal correction was achieved with multilevel Ponte osteotomies. The patient's postoperative course was uncomplicated, and he was discharged home 5 days after surgery with no neurological deficits.

For 5 months, the patient had significant improvement

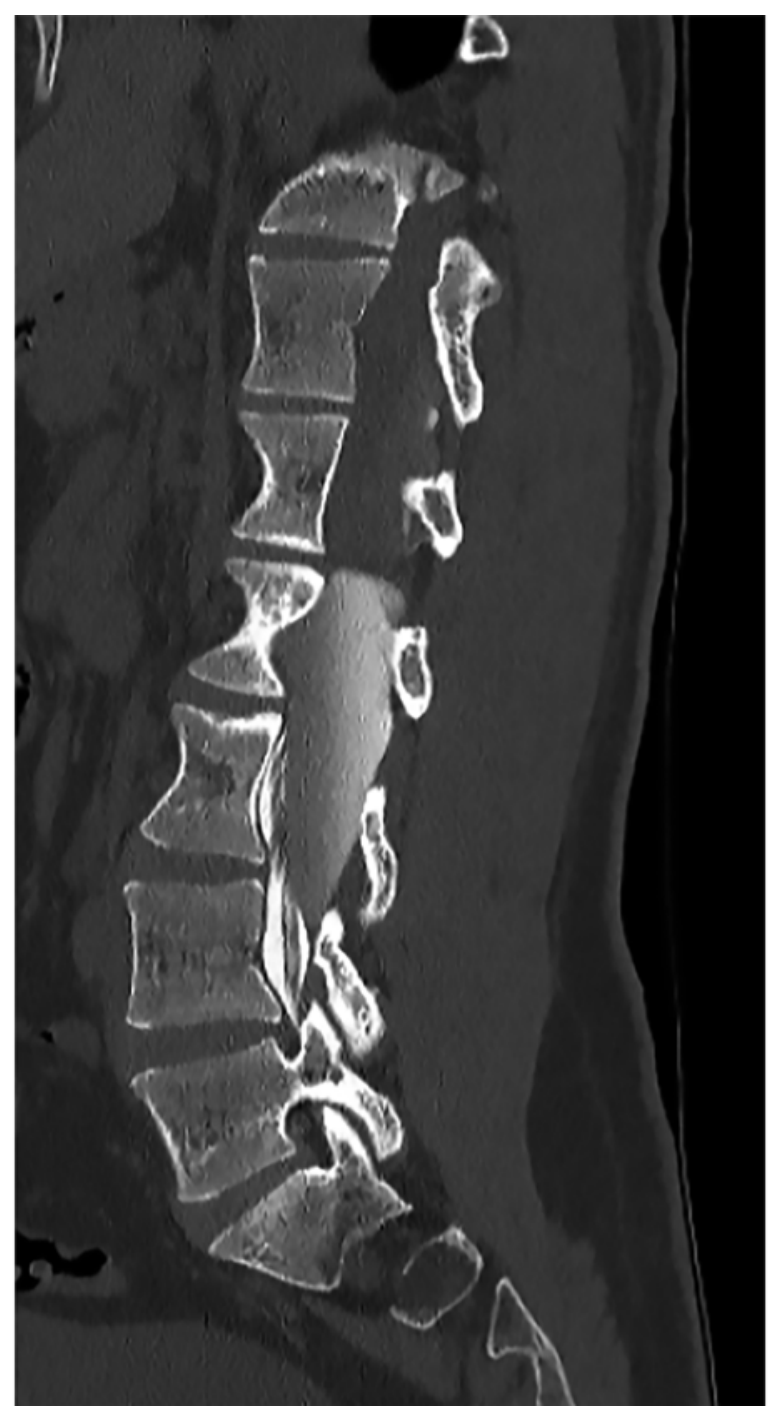

FIG. 2. Sagittal CT myelogram of arachnoid cyst spanning T-12 to L-4.

in his back pain and enjoyed full activity. He returned to his hometown and planned to follow up in 1 year. $\mathrm{He}$ then experienced an insidious neurological decline over a 4-month period. When he returned to our clinic 9 months postoperatively, he was diagnosed with cauda equina syndrome. He had full strength in his proximal lower extremities, but only $2 / 5$ strength with dorsiflexion bilaterally. $\mathrm{He}$ had diminished sensation in his perianal region and experienced sexual dysfunction.

Magnetic resonance imaging revealed an unchanged arachnoid cyst spanning T-12 to L-4 that anteriorly displaced the conus medullaris and cauda equina (Fig. 4). CT imaging revealed no encroachment of the neural elements by any of the pedicle screws. The patient then had an electromyogram, which suggested cauda equina syndrome. His neurological status led us to recommend lumbar decompression with fenestration of the cyst. Because of suboptimal positioning of the inferior lumbar pedicle screws and residual lumbar scoliosis with obliquity at the L3-4 disc space, we also planned an extension of the fusion to L-4.

The T3-L3 fixation and fusion were extended to L-4 

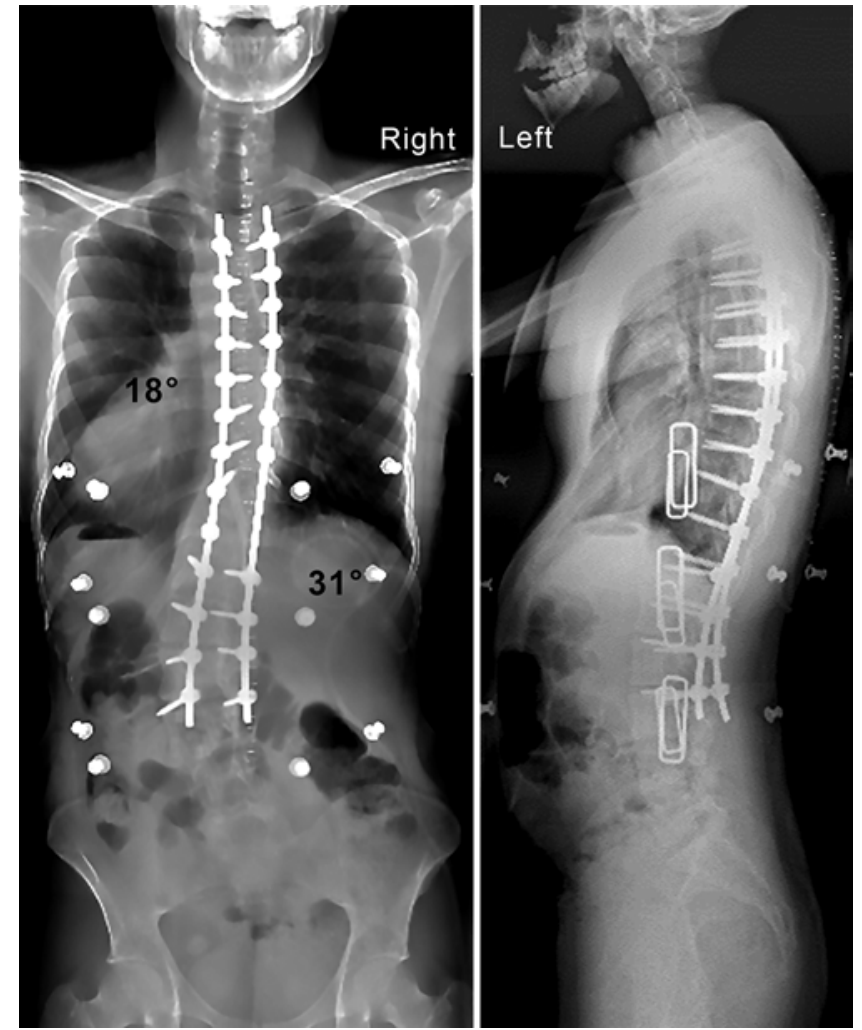

FIG. 3. Postoperative AP (left) and lateral (right) radiographs. External artifacts are from a thoracolumbosacral orthosis, a corset-style brace designed to restrict movement of the torso, such as after surgery.

and connected to the existing construct (Fig. 5). The dorsal fusion mass and laminae from T-12 through L-4 were removed using a high-speed drill and Kerrison rongeurs. After performing the laminectomies, we brought in the operative microscope and observed an extremely thin and dysplastic dural layer. In many large areas, the dura appeared to be completely absent. The entire defect was approximately $15 \mathrm{~cm}$ long. We fenestrated a pressurized arachnoid compartment spanning L-2 to L-4. We then identified several additional arachnoid compartments adjacent to the L2-4 compartment. Using microsurgical techniques, we thoroughly fenestrated each compartment, ensuring that the lumbosacral nerve roots were decompressed and communication of CSF was restored in the cranial and caudal directions.

After the arachnoid fenestration, there were only scant remnants of friable dural edges. There was insufficient quantity and quality of dura to close the defect with primary closure or a dural patch graft. Therefore, we placed a collagen onlay graft, DuraGen (Integra LifeSciences Corp.), over the exposed nerve roots. The edges of the graft were tucked beneath the edges of the remaining lamina and facets. A thin layer of a hydrogel sealant, DuraSeal (Integra LifeSciences Corp.), was applied over the collagen graft. One additional layer of the collagen graft and sealant were then placed. The wound was then closed in a meticulous multilayer fashion, as it had been previously, by using Vicryl sutures (Ethicon US, LLC). The skin was closed with a running nylon suture.

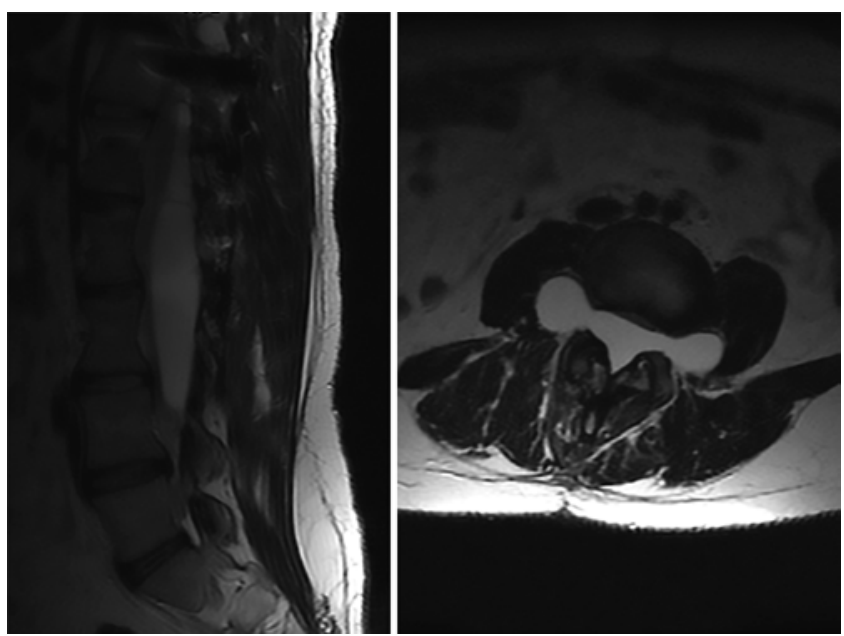

FIG. 4. Sagittal (left) and AP (right) MR images of thoracolumbar arachnoid cyst demonstrating anterior displacement of nerve roots and extension out of the foramen bilaterally.

Approximately 3 weeks later, the patient returned to the emergency department with severe positional headaches and a large pseudomeningocele at the incision site. He consented to an exploration of his wound with attempted reconstruction of his posterior elements by using a novel technique with titanium mesh and a methylmethacrylate (MMA) cast.

After reopening the incision, we evacuated a large col-
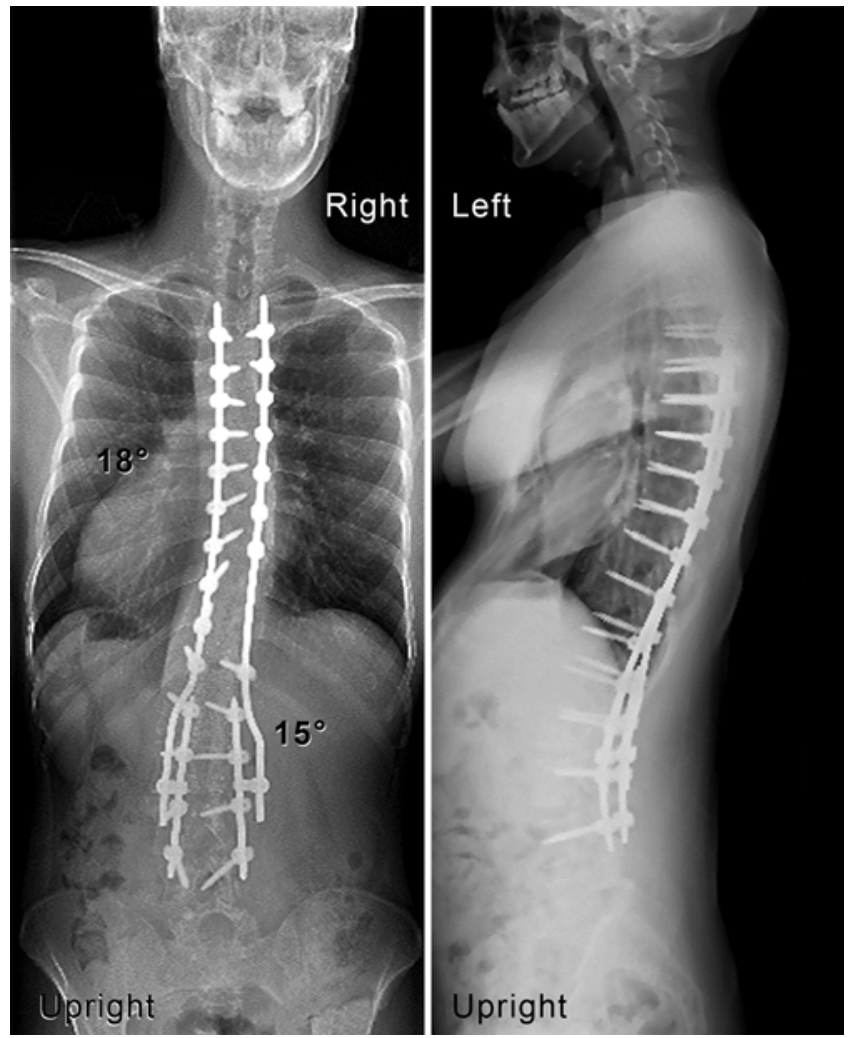

FIG. 5. Postoperative AP (left) and lateral (right) radiographs obtained 1 month after lumbar decompression and extension of fusion. 

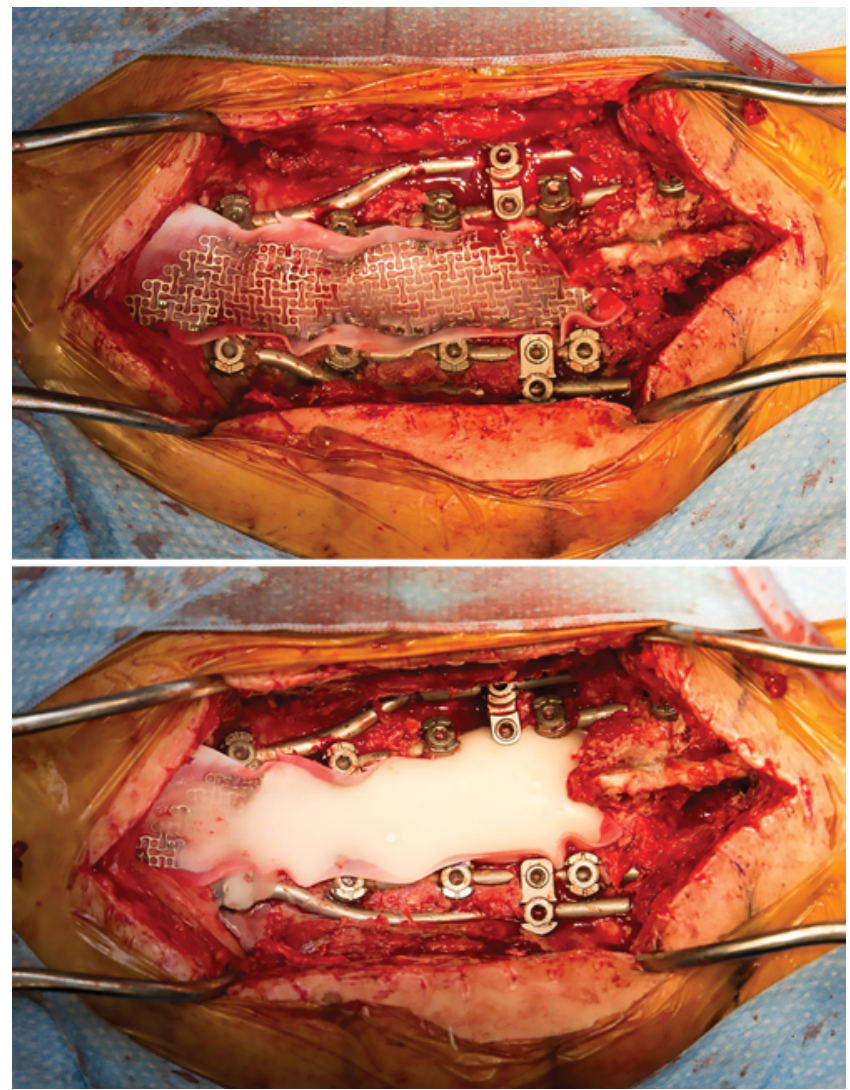

FIG. 6. Intraoperative photographs obtained after insertion of titanium mesh scaffold (upper) and after insertion of MMA cast (lower).

lection of CSF from the pseudomeningocele. The collagen graft was degraded and CSF was leaking through several sites. The edges of the laminectomy defect were exposed with monopolar cautery. An additional piece of collagen graft was placed over the defect. We then molded a piece of titanium mesh into a slightly curved, hemitubular configuration and secured it over the entire laminectomy defect with multiple 5-mm titanium screws (Fig. 6 upper). Care was taken to ensure that the mesh was shaped in a fashion that did not compress the thecal sac and the neural elements. The area under the mesh was probed with a dental instrument to verify that there was no compression. The mesh was then reinforced with an MMA layer, and $1 \mathrm{~g}$ vancomycin powder was mixed with the MMA cement prior to hardening (Fig. 6 lower). The collagen graft under the titanium mesh plate served as a barrier to the thecal sac, preventing any MMA from penetrating the mesh and adhering to neural elements. A Valsalva maneuver was then performed and no egress of CSF was observed. A lumbar drain was inserted at the L4-5 interspace and tunneled out through a separate stab incision. The wound was closed in layers with Vicryl sutures, and the skin was closed with a running nylon suture. The patient's lumbar drain was maintained for 5 days of CSF drainage, and he was discharged without new neurological deficits 8 days after this novel surgery.

At his 24-month postoperative follow-up, the patient had recovered full strength in his legs, and his sensory deficits and sexual dysfunction had resolved. His incision had healed well, with no signs of pseudomeningocele. He had no additional positional headaches.

\section{Discussion}

This report highlights the unique case of a young man with an asymptomatic thoracolumbar arachnoid cyst who underwent a successful operation to correct a spinal deformity. Unfortunately, he experienced a delayed neurological decline. Although the exact mechanism that led to the decline is unknown, we suspect that the CSF flow pattern within the arachnoid cyst was altered after surgery. We believe that either a single compartment or multiple compartments within the arachnoid cyst started to entrap CSF through a 1-way valve effect and enlarged the cyst. This enlargement led to progressive compression of the lumbosacral nerve roots and to cauda equina syndrome.

Because of his neurological decline, we elected to fenestrate the compartments within the cyst and to decompress the nerve roots. We resected as much of the cyst wall as possible and ensured good CSF flow rostrally and caudally. Given the patient's large dural defects, we initially planned to reconstitute the dural layer, which included 2 layers of collagen onlay graft and hydrogel sealant.

When the patient returned several weeks later with an incisional pseudomeningocele and intractable headaches, we decided to use a different strategy to reconstitute the dural layer. To our knowledge, there are no techniques described in the literature for managing such a large spinal dural defect. We adopted principles of dural reconstruction with multilayer closure, similar to those used in skull base reconstruction. ${ }^{6,8}$

We reconstructed the posterior elements with titanium mesh fortified with an MMA layer. This reconstruction was critical to the successful outcome because it eliminated the dead space created by the laminectomy defects and provided a barrier to CSF egress. It also provided a tamponade effect on the collagen graft during periods of thecal sac expansion and high CSF pressure.

Several key nuances characterize this technique. The titanium mesh plate must be molded in a shape that does not compress the thecal sac and the neural elements. After placement of the titanium mesh, it is important to verify that it is not causing any stenosis. It is also imperative to place a layer of collagen graft over the thecal sac before placing the MMA over the mesh. This barrier to the thecal sac prevents MMA from contacting and adhering to the nerves. Because of the revision in this case and the implantation of a foreign body, we mixed vancomycin powder with the MMA cement before placing it over the titanium mesh, in an effort to prevent a gram-positive infection. As with any closure involving dural repair, the key to a successful outcome requires elimination of the dead space, with approximation of the paraspinal musculature and a meticulous watertight fascial closure.

The role of a lumbar drain in the management of this patient's dural defect is certainly debatable. During the first dural repair, we elected not to place a lumbar drain for several reasons. First, we initially believed that the DuraGen collagen onlay graft and the dural sealant, in combi- 
nation with a watertight fascial closure, would be adequate to prevent a CSF leak. Second, a lumbar drain is not without risks, including the risk of infection and overdrainage. In hindsight, we believe that the initial repair failed because it was inadequate due to the size of the defect and the friable nature of the dural edges; it is unlikely that the placement of a lumbar drain would have changed the initial outcome. However, in the second repair, a lumbar drain was placed (despite its risks) because of the failed first repair. The CSF diversion probably facilitated healing during its temporary use; however, the success of the second repair was dependent on recreating the posterior elements with MMA and a titanium mesh plate.

\section{Conclusions}

To our knowledge, this is the first report to describe a multilayer closure technique in which a collagen graft, titanium mesh, and MMA were used to reconstitute the dural compartment in the spine. The patient's defect was successfully managed with this technique. Although this is not a first-line strategy for dural closure in the spine, this technique can be considered in challenging cases with large dural defects that are not amenable to closure with traditional techniques.

\section{Acknowledgments}

We thank the staff of the Neuroscience Publications office at Barrow Neurological Institute for their support.

\section{References}

1. Clajus C, Stockhammer F, Rohde V: The intra- and postoperative management of accidental durotomy in lumbar spine surgery: results of a German survey. Acta Neurochir (Wien) 157:525-530, 2015

2. da Costa LB, Ahn H, Montanera W, Ginsberg H: Repeated meningitis as a delayed complication of scoliosis surgery. $\mathbf{J}$ Spinal Disord Tech 20:333-336, 2007

3. Dafford EE, Anderson PA: Comparison of dural repair techniques. Spine J 15:1099-1105, 2015

4. Jeon SH, Lee SH, Tsang YS, Jung TG, Moon KH, Choi G, et al: Watertight sealing without lumbar drainage for incidental ventral dural defect in transthoracic spine surgery: a retrospective review of 53 cases. J Spinal Disord Tech 30:E702E706, 2017

5. Kim KD, Wright NM: Polyethylene glycol hydrogel spinal sealant (DuraSeal Spinal Sealant) as an adjunct to sutured dural repair in the spine: results of a prospective, multicenter, randomized controlled study. Spine (Phila Pa 1976) 36:1906-1912, 2011

6. Kusumi M, Fukushima T, Aliabadi H, Mehta AI, Noro S, Rosen CL, et al: Microplate-bridge technique for watertight dural closures in the combined petrosal approach. Neurosurgery 70 (2 Suppl Operative):264-269, 2012

7. Papavero L, Engler N, Kothe R: Incidental durotomy in spine surgery: first aid in ten steps. Eur Spine J 24:2077-2084, 2015

8. Sekhar LN, Sarma S, Morita A: Dural reconstruction with fascia, titanium mesh, and bone screws: technical note. Neurosurgery 49:749-752, 2001

9. Wright NM, Park J, Tew JM, Kim KD, Shaffrey ME, Cheng J, et al: Spinal sealant system provides better intraoperative watertight closure than standard of care during spinal surgery: a prospective, multicenter, randomized controlled study. Spine (Phila Pa 1976) 40:505-513, 2015

\section{Disclosures}

The authors report no conflict of interest concerning the materials or methods used in this study or the findings specified in this paper.

\section{Author Contributions}

Conception and design: all authors. Acquisition of data: all authors. Analysis and interpretation of data: all authors. Drafting the article: all authors. Critically revising the article: all authors. Reviewed submitted version of manuscript: all authors. Statistical analysis: all authors. Administrative/technical/material support: all authors. Study supervision: Kakarla.

\section{Correspondence}

U. Kumar Kakarla, c/o Neuroscience Publications, Barrow Neurological Institute, St. Joseph's Hospital and Medical Center, 350 W Thomas Rd., Phoenix, AZ 85013.email: neuropub@ dignityhealth.org. 